\title{
Uma proposta de design de informação para as novas curvas de ganho de peso gestacional da caderneta da gestante no Brasil
}

\author{
An information design proposal for the new Brazilian gestational weight gain charts \\ in the pregnant woman's booklet
}

\author{
Bruno Augusto Lorenz, Letícia Ange Pozza, Thaís Rangel Bousquet Carrilho, \\ Gilberto Kac, Francisco Estivallet
}

ganho de peso gestacional, design de informação, caderneta da gestante.

Toda mulher grávida precisa ganhar peso ao longo de sua gestação. O ganho de peso é um dos principais indicadores utilizados para monitorar o estado nutricional da mãe e, por consequência, sua saúde e a do feto durante a gestação. Este indicador pode ser acompanhado por meio de um gráfico, no qual o eixo horizontal representa o número de semanas de gestação, e o eixo vertical indica os valores de ganho de peso da gestante. Atualmente, o monitoramento do estado nutricional no Sistema Único de Saúde (SUS) brasileiro é feito por meio de um gráfico de índice de massa corporal (IMC), construído para gestantes chilenas e que não reflete a realidade das mulheres brasileiras. Desta forma, criar um gráfico para monitoramento do ganho de peso gestacional no SUS é necessário. O presente artigo descreve um estudo de caso sobre o design do gráfico destas novas curvas de ganho de peso. Apresentam-se os estudos construídos, as atividades de discussão junto aos profissionais do Ministério da Saúde e os aprendizados obtidos ao longo do processo: a) exploração do design de informação e do desenvolvimento de protótipos enquanto meio de democratização da ciência; b) amplificação do acesso das curvas por meio de novos formatos e; c) reconhecimento da importância de processos de co-design para projetos de design de informação.

gestational weight gain, information design, pregnant woman's booklet.

Every pregnant woman needs to gain weight throughout her pregnancy. Weight gain is one of the main indicators used to monitor the mother's nutritional status and, consequently, her health and that of the fetus during pregnancy. This index can be checked in a graph, in which the horizontal axis represents the number of weeks of gestation, and the vertical axis indicates the pregnant woman's weight gain values. Currently, the monitoring of nutritional status in the Brazilian Unified Health System (SUS) is done through a body mass index (BMI) chart, built for Chilean pregnant women and that does not reflect the reality of Brazilian women. Thus, creating a chart for monitoring gestational weight gain in SUS is necessary. This article describes a case study on the graphic design of these new weight gain curves. The constructed studies, discussion activities with professionals from the Ministry of Health and the lessons learned throughout the process are presented: a) exploration of information design and the development of prototypes as a means of democratizing science; $b$ ) amplification of access to curves through new formats and; c) recognition of the importance of co-design processes for information design projects.

Anais do $10^{\circ} \mathrm{CIDI}$ e $10^{\circ} \mathrm{CONGIC}$

Kelli C.A.S. Smythe, Rafael de Castro Andrade (orgs.)

Sociedade Brasileira de Design da Informação - SBDI

Curitiba | Brasil | 2021
Proceedings of the $10^{\text {th }} \mathrm{CIDI}$ and $10^{\text {th }}$ CONGIC

Kelli C.A.S. Smythe, Rafael de Castro Andrade (orgs.)

Sociedade Brasileira de Design da Informação - SBDI Curitiba | Brazil | 2021 


\section{Introdução}

Toda mulher grávida precisa ganhar peso ao longo de sua gestação. O ganho de peso é um dos principais indicadores utilizados para monitorar o estado nutricional da mãe e, por consequência, sua saúde e a do feto durante a gestação. Este indicador pode ser acompanhado por meio de um gráfico, no qual o eixo horizontal representa o número de semanas de gestação, e o eixo vertical indica os valores de ganho de peso da gestante.

Desde a década de 1980, o Ministério da Saúde utiliza ferramentas para monitoramento do estado nutricional na gestação que não foram criadas para mulheres brasileiras (Kac e Carrilho et al., 2021). Enquanto a curva de Atalah et al. (1997) possui baixa capacidade de predizer desfechos infantis adversos, as recomendações do Instituto de Medicina Americano (Institute of Medicine, 2009) não foram testadas e validadas no Brasil, refletindo apenas a realidade das gestantes norte-americanas. Um estudo de 2009 (Kac et al. 2009), por exemplo, apontou que as curvas de Atalah são inadequadas para predizer a ocorrência de baixo peso ao nascer e de bebês Pequenos para a Idade Gestacional (PIG) ou Grandes para a Idade Gestacional (GIG) (Kac et al., 2021).

Desde 2005, um grupo de pesquisadores brasileiros liderados pelo pesquisador Gilberto Kac discute a necessidade da criação de novas curvas e recomendações para gestantes brasileiras. A pesquisa intitulada "Proposição de novas recomendações de ganho de peso gestacional para utilização do SUS" é uma iniciativa do Observatório de Epidemiologia Nutricional Universidade Federal do Rio de Janeiro (UFRJ) apoiada pela Coordenação Geral de Alimentação e Nutrição do Ministério da Saúde (MS), que tem como objetivo principal a criação de um novo instrumento para monitoramento do ganho de peso gestacional e de novas recomendações baseadas neste instrumento.

As curvas propostas por Kac e Carrilho et al. (2021) foram construídas com base em dados de 21 estudos brasileiros, realizados entre os anos de 1990 e 2018, que compõem a base de dados do Consórcio Brasileiro de Nutrição Materno-infantil (CONMAI) (Carrilho et al., 2020). O conjunto de dados utilizado na construção das curvas incluiu 7.086 mulheres brasileiras com 29.323 medidas repetidas de ganho de peso.

Na figura 1, é possível observar as quatro curvas criadas - uma para cada categoria de IMC: Baixo Peso (IMC $<18,5 \mathrm{~kg} / \mathrm{m}^{2}$ ), Eutrofia (IMC $\geq 18,5 \mathrm{e}<25 \mathrm{~kg} / \mathrm{m}^{2}$ ) Sobrepeso (IMC $\geq 25 \mathrm{e}$ $<30 \mathrm{~kg} / \mathrm{m}^{2}$ ) e Obesidade (IMC $\geq 30 \mathrm{~kg} / \mathrm{m}^{2}$ ). Os métodos utilizados na construção destas curvas estão descritos em Kac e Carrilho et al. (2021). 
Figura 1 - Novas curvas de ganho de peso gestacional (Fonte: autoria própria)
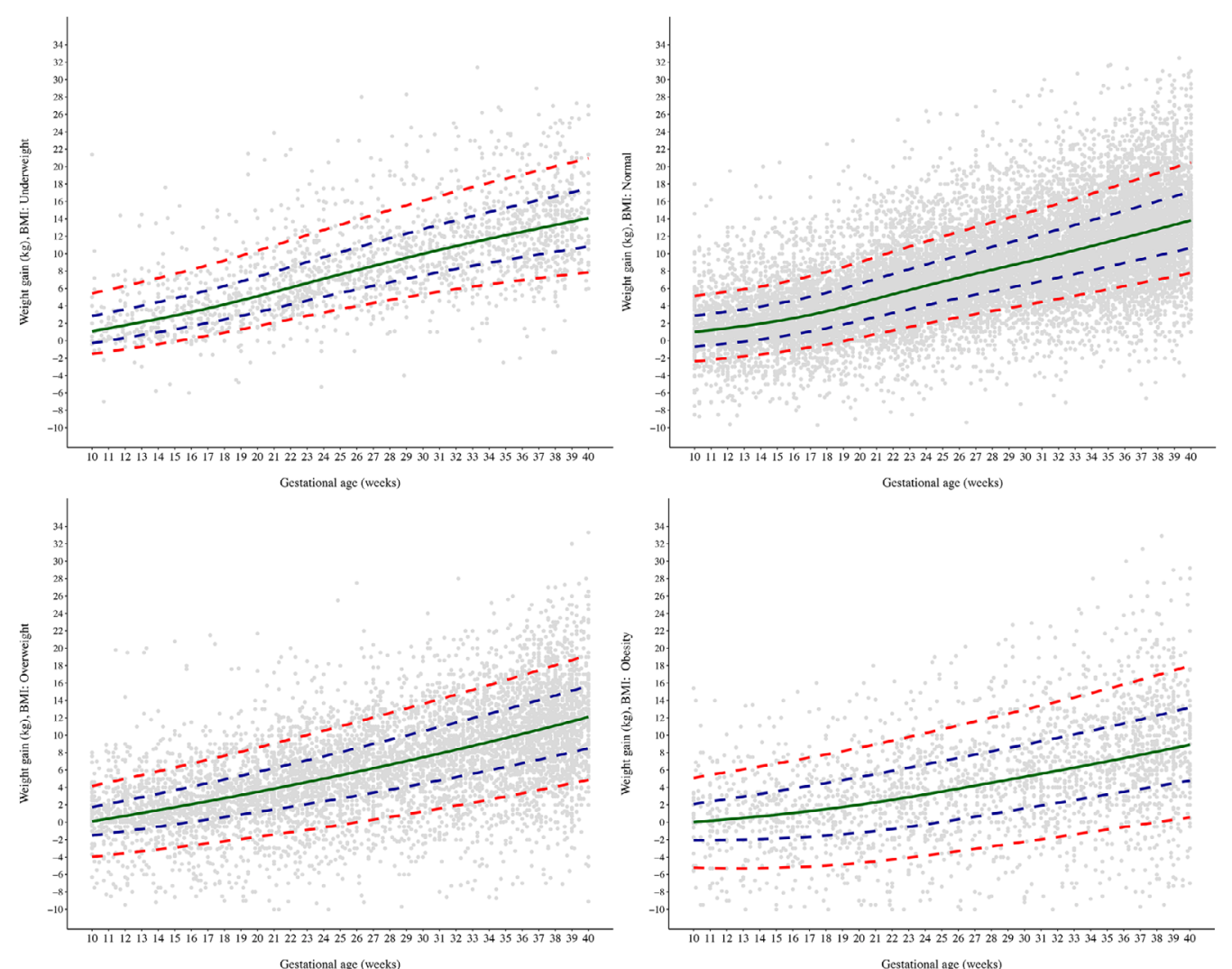

Para utilizar o instrumento, primeiramente o IMC da gestante deve ser calculado na primeira consulta de acompanhamento pré-natal. Feita essa mensuração, a gestante se encaixa em uma das quatro categorias de IMC, a partir daí, ela deve acompanhar o ganho de peso de acordo com as indicações da categoria correspondente, considerando os percentis equivalentes (indicados na imagem acima pelas linhas coloridas: Vermelhas: P10 e P90; Azuis: P25 e P75; Verde: Mediana (P50).

Atualmente, a ferramenta de acompanhamento de curvas de ganho de peso, utilizado pelo Sistema Único de Saúde (SUS) brasileiro, está disponível na Caderneta da Gestante ${ }^{1}$ (observar a figura 2, que apresenta o gráfico da atual caderneta). Esse material apresenta informações que auxiliam e orientam a mulher ao longo de toda a gestação, sendo impresso e distribuído anualmente pelo Ministério da Saúde (MS).

O gráfico da curva utilizado neste material, no entanto, apresenta dois problemas principais: i) ele foi desenvolvido considerando as mensurações internacionais, que não abrangem dados de mulheres brasileiras e, ii) a visualização de dados não possibilita uma observação confiável do ganho de peso ao longo do tempo, pois desconsidera que cada uma das categorias de IMC possui curvas diferentes de ganho entre si.

\footnotetext{
${ }^{1}$ Disponível em <https://portalarquivos2.saude.gov.br/images/pdf/2018/agosto/31/Caderneta-da-Gestante-2018.pdf>. Acesso em 9 de maio de 2021.
} 
Gráfico de acompanhamento nutricional

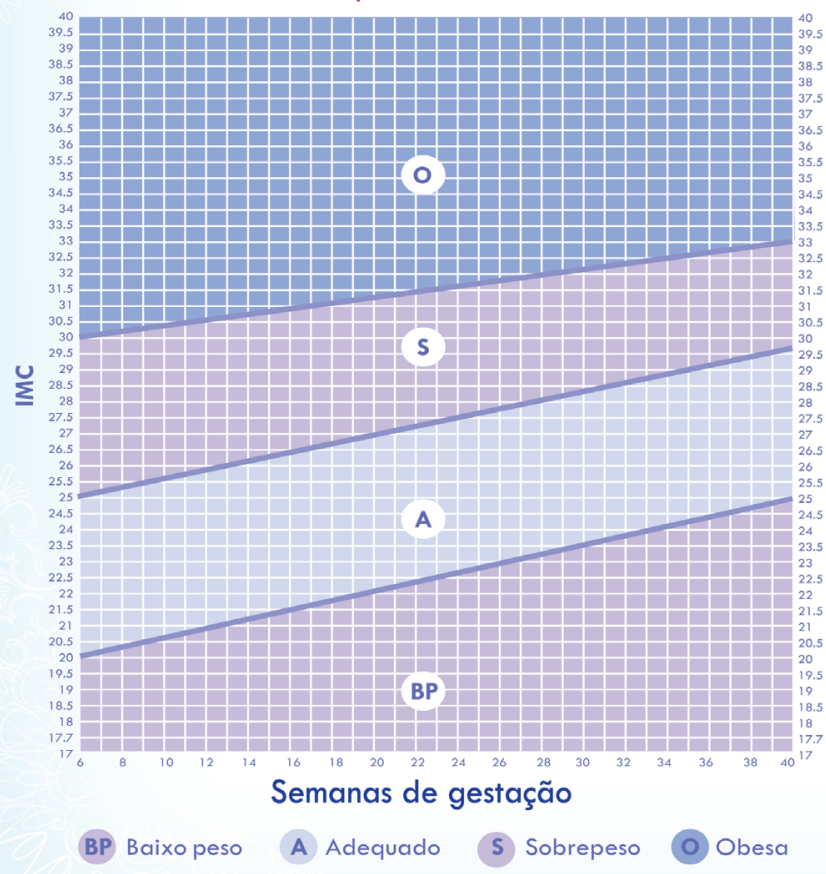

Se estabelece, assim, o problema de design enfrentado pelos designers envolvidos no projeto: adaptar os gráficos de ganho de peso gestacional a partir das novas curvas geradas pelos pesquisadores, considerando os problemas identificados na curva atual e o espaço disponível na caderneta da gestante, envolvendo as áreas técnicas do MS e os profissionais de saúde que irão utilizar o material no acompanhamento das gestantes.

Por meio de um exercício de research through design, que considera o desenvolvimento de protótipos como parte essencial do processo de pesquisa e na construção do conhecimento (Koskinen et al., 2011; Gaver, 2012), o redesenho das curvas abriu oportunidades para facilitar debates sobre o uso da ferramenta por gestantes e técnicos de saúde pública, tanto para preenchimento quanto para a interpretação dos dados e também questionar sobre o espaço dedicado à visualização na caderneta da gestante do SUS.

O artigo se divide em três seções: 1) Apresentação dos processos de desenvolvimento, reflexões e decisões acerca das visualizações, bem como a influência dos demais atores, possibilitada por meio de oficinas de validação; 2) discussão sobre os desafios e aprendizados alcançados e, por fim; 3) considerações finais.

\section{Processo e Desenvolvimento}

O trabalho da criação do gráfico junto aos pesquisadores compreendeu um processo de projeto com etapas de briefing, geração de alternativas e rodadas de validação interna (entre as equipes de design e de pesquisa), além de rodadas de validação externa (junto à técnicos do MS e de profissionais de atenção básica à saúde do SUS). Uma síntese de tal processo pode ser conferida na figura 3 . 
Figura 3: Etapas do processo de design (Fonte: autoria própria)

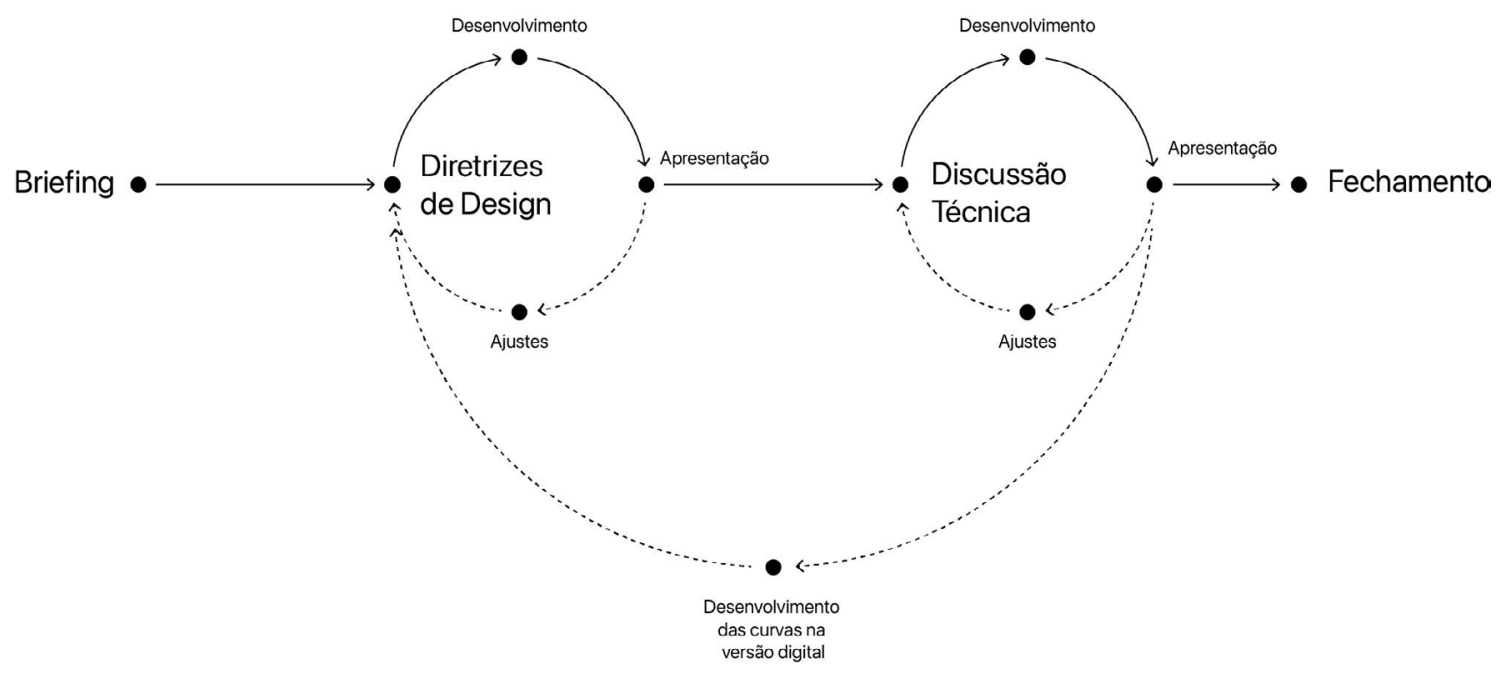

Desde o início do trabalho foi estabelecida uma relação de colaboração entre as equipes de design e de pesquisa, em que ambas trabalharam juntas nos processos de projeto, e acolhendo os não-designers como especialistas em suas realidades e parceiros valiosos nos debates e descobertas sobre questões fundamentais do projeto (Sanders, Staples, 2008).

$\mathrm{Na}$ reunião inicial de briefing, foram estabelecidas as primeiras diretrizes de projeto: i) foco na leitura e na compreensão das informações tanto por parte das gestantes quanto dos profissionais de saúde; ii) explorar formas de inserção dos dados da gestante na curva, considerando que essa é uma ferramenta de acompanhamento e esses dados devem ser preenchidos ao longo do tempo; iii) considerar diferentes cegueiras cromáticas que podem prejudicar a leitura do gráfico (daltonismo, por exemplo). Desde o início, emergiu o interesse de ambas as partes na construção de protótipos tanto da versão impressa quanto da versão digital das curvas que poderiam ser adotados pelo MS, por pesquisadores do meio acadêmico e por instituições privadas em diversos âmbitos.

Ao entender que um dos principais entregáveis seria a versão impressa, foi preciso atentar imediatamente sobre o espaço reservado para o instrumento e sobre o contexto da aplicação na caderneta do SUS. Na figura 4, é possível ver a página que apresenta a curva de Atalah e os espaços disponíveis: 
Figura 4 - Layout da página atual e suas medidas (Fonte: Caderneta da gestante do SUS)
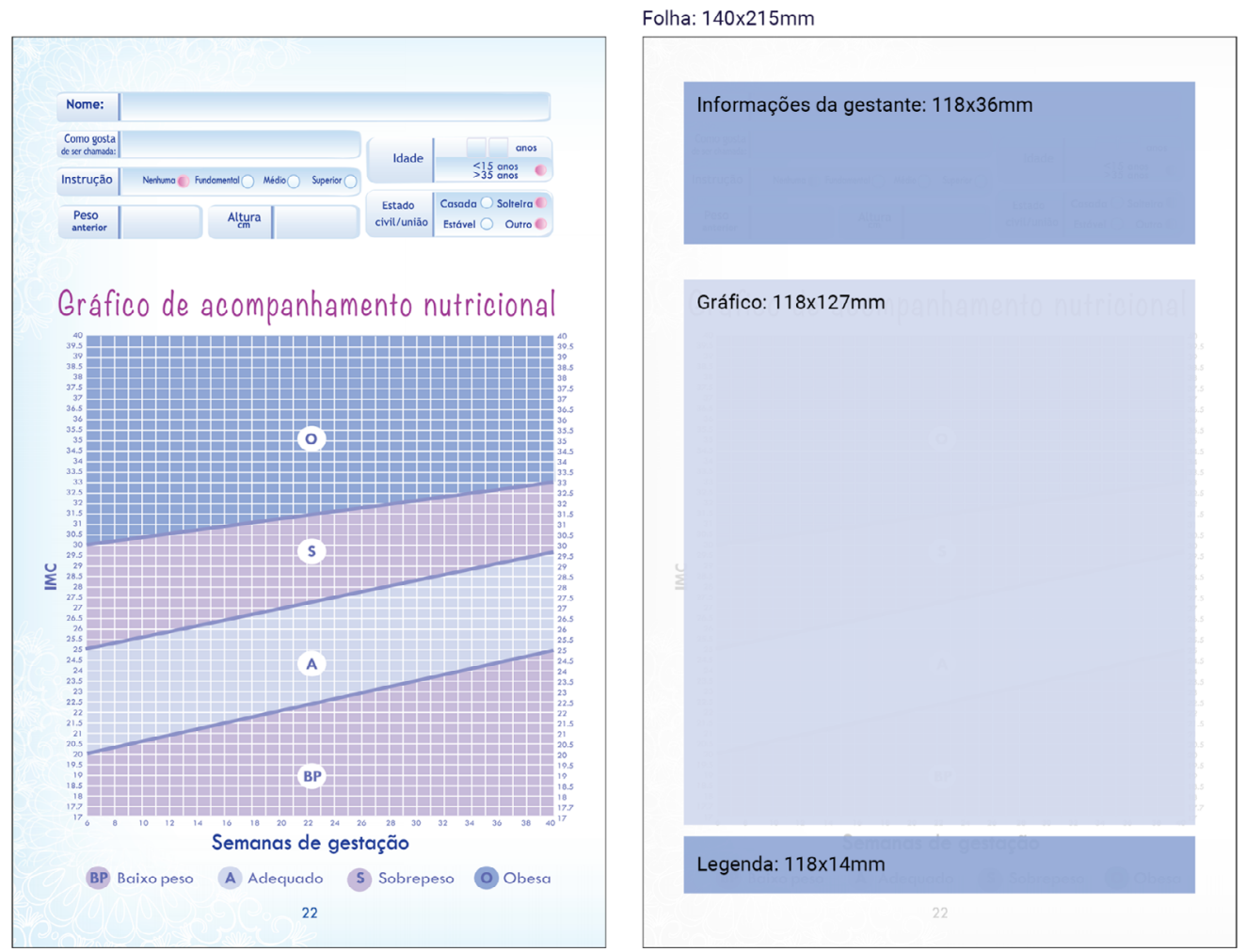

Logo nos primeiros estudos, a equipe de designers identificou que a proposta atual da curva era ambígua em seu preenchimento ("Deve-se preencher dentro dos quadrados ou em cima da linha?"). Inspirados pela síntese gráficas clássicas de mapas como o do metrô britânico criado por Harry Beck in $1931^{2}$ (detalhe em A, na figura 5), os designers propuseram substituir a grade atual por outra que apresentava pequenos círculos que indicariam o preenchimento das informações de maneira mais direta, estratificando também o eixo vertical do IMC em intervalos de $500 \mathrm{gr} / \mathrm{m}^{2}$ (B, na figura 5). O próximo passo foi o de inserir as quatro faixas de ganho de peso sobre a graficação e, em seguida, explicitar o range de cada uma das faixas de peso com cores (C, D, E), como é possível visualizar na figura 5 .

\footnotetext{
2 Disponível em <https://tfl.gov.uk/corporate/about-tfl/culture-and-heritage/art-and-design/harry-becks-tube-map>. Acesso em 13 de maio de 2021.
} 
Figura 5 - Estudos Iniciais (Fonte: autoria própria)

A

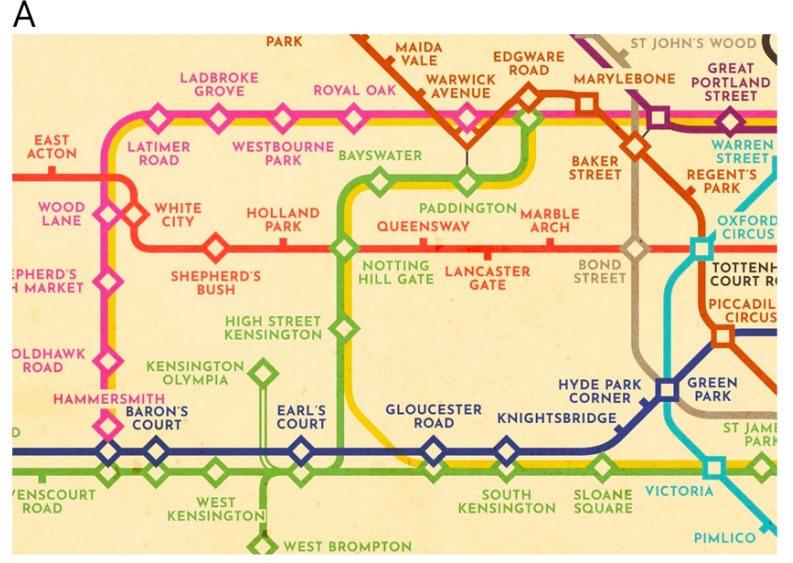

C

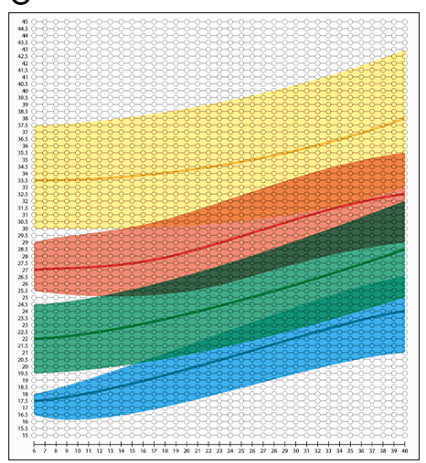

D

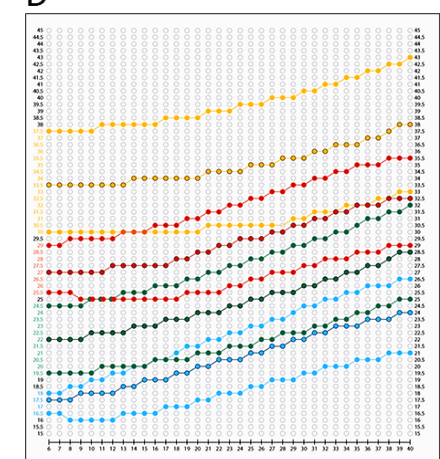

B

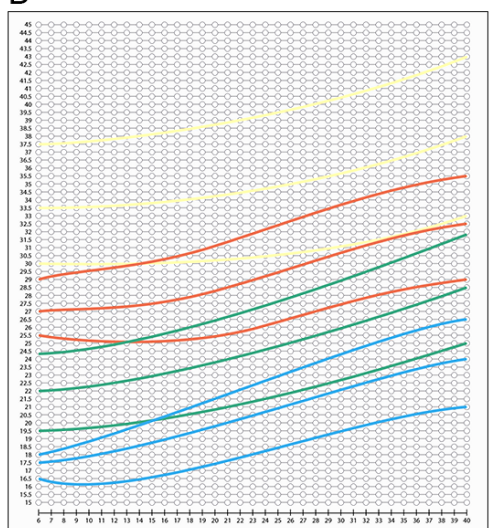

$\mathrm{E}$

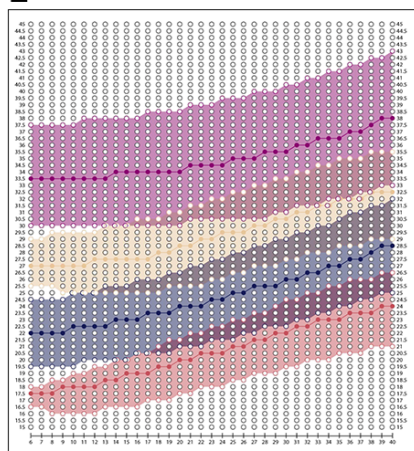

Com essa primeira bateria de testes e experimentações gráficas, os designers perceberam que a sobreposição das curvas poderia dificultar a compreensão dos dados. Essa sobreposição era essencial, considerando o espaço disponível na página da caderneta da gestante. Desse modo, tentou-se estabelecer uma paleta de cores categórica que servisse para diferenciar as faixas de peso entre si. Uma paleta foi escolhida e testes de cegueira cromática foram realizados utilizando a ferramenta Coblis ${ }^{3}$ (figura 6).

Figura 6 - Testes de cegueira cromática (Fonte: autoria própria)
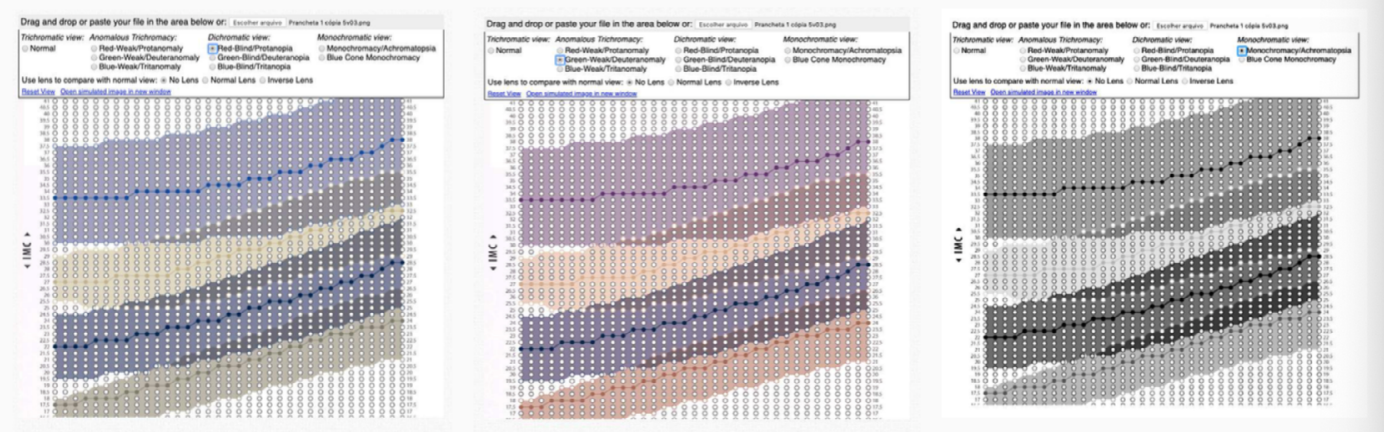

${ }^{3}$ Disponível em <https://www.color-blindness.com/coblis-color-blindness-simulator/>. Acesso em 9 de maio de 2021. 
Tendo em mãos esses primeiros experimentos, os designers reuniram-se com os pesquisadores para a primeira sessão de avaliação interna. A ideia central estabelecida arredondar os valores do IMC e utilizar as bolinhas como recurso gráfico para facilitar o preenchimento das informações - não foi aprovada pois os pesquisadores entenderam que essa síntese poderia afetar os diagnósticos de ganho ou perda de peso. Isso porque o ganho de peso de uma gestante deve ser constante e positivo, de tal forma que toda semana ela pese mais do que na semana anterior, e a síntese gráfica proposta pelos designers não sugeria tal variação.

Essa sugestão, no entanto, foi útil ao debate pois ambas as equipes refletiram sobre a necessidade de questionar a granularidade do eixo vertical e a possibilidade apresentar outras grandezas nesse eixo para identificar a variação: i) o peso da gestante e ii) o ganho de peso da gestante uma consulta para outra. Os pesquisadores observaram também que há casos em que a gestante não fica "dentro das curvas" de ganho de peso e, para isso, seria necessário avaliar os limites aceitáveis de máximo e mínimo do eixo vertical.

Após o descarte da proposta inspirada pelos mapas metroviários, ambos os grupos decidiram que a alternativa a ser considerada seria a indicação da demarcação sobre as linhas do gráfico do peso da gestante em relação à semana de gravidez que ela se encontrava. Desse modo, foi necessário voltar ao grid formado por linhas horizontais e verticais para facilitar o preenchimento desses dados. Para a próxima rodada de estudos, os designers preocuparam-se em estudar formas de preenchimento das informações no gráfico (figura 7) e as possibilidades de implementação das curvas considerando o espaço disponível na caderneta da gestante (figura 8). 
Figura 7 - testes de preenchimento (Fonte: autoria própria)

1

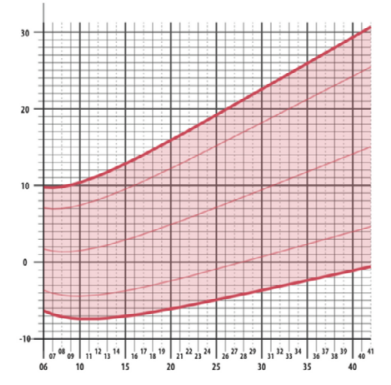

4

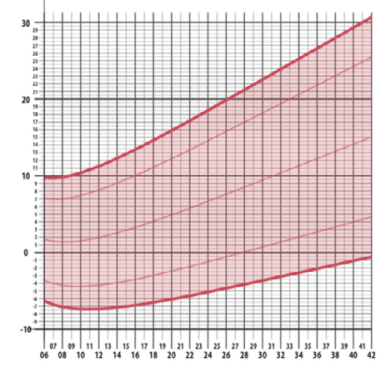

2

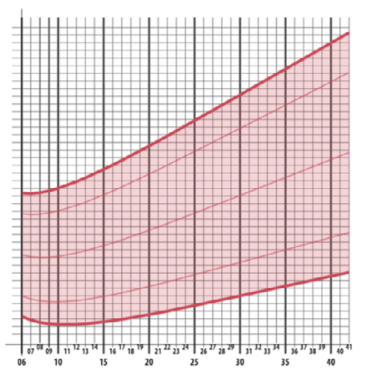

5

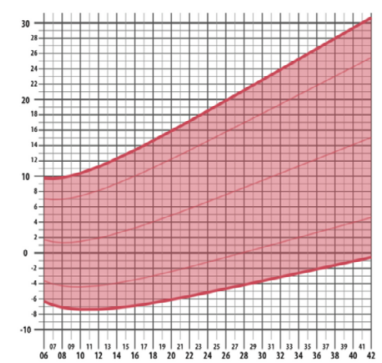

3

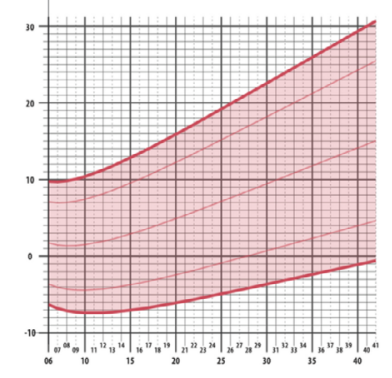

6

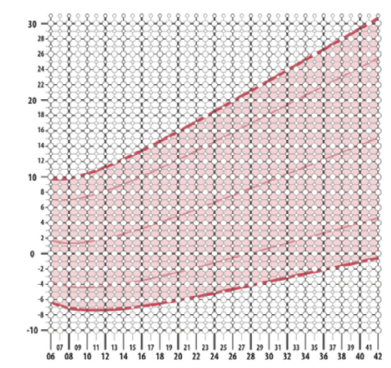

Durante a prototipação ficou claro que uma atenção especial deveria ser dada às decisões relacionadas à tipografia - principalmente no que diz respeito ao tamanho e peso das fontes escolhidas, o tamanho da página da caderneta da gestante e a boa legibilidade dessas informações. Assim, a família tipográfica Oswald foi escolhida, devido ao número de pesos disponíveis e pelo seu desenho condensado sem serifas que permitiu que os intervalos das escalas dos eixos horizontal e vertical fossem inseridos com clareza.

Uma das diretrizes de projeto estabelecidas nesta segunda rodada de estudos dizia respeito ao número ideal de semanas de gravidez representadas no eixo horizontal do gráfico, sendo necessária uma tomada de decisão tanto sobre o número inicial quanto o número final de semanas representadas na visualização. O eixo das semanas não poderia se iniciar na semana 1 pois, de acordo com a equipe de pesquisadores, as primeiras manifestações da gravidez são percebidas apenas a partir da sexta semana de gestão - o que torna incomum uma coleta de dados do peso antes desse período. Com relação ao outro extremo do eixo, os pesquisadores estabeleceram que, naquele momento, o número de semanas deveria ir até o número 42 , tendo em vista que há casos de gestação pós-termo - quando a gestação passa das habituais 40 semanas de gravidez.

Foram exploradas alternativas diversas de representação desse intervalo: apenas os números pares eram representados numericamente; todos os números representados, mas apenas os pares como número maior; e demarcações com linhas mais grossas para sinalizar graficamente a passagem do tempo e facilitar o preenchimento dos valores quando o dado inserido não estava próximo dos numerais. 
Outro aspecto importante a ser explorado a partir das discussões dos primeiros estudos foi o da sobreposição das curvas em um ou dois gráficos ou a construção de gráficos menores, mas separados. Por um lado, essa última proposta facilitaria a leitura e a inserção dos dados, já que seria mais claro ao profissional e à gestante qual seria sua faixa de peso já na primeira consulta. Por outro lado, poderia prejudicar a legibilidade pois o espaço necessário para as informações seria ainda mais exíguo, como pode-se conferir na Figura 8.

Figura 8 - Testes considerando o espaço disponível (Fonte: autoria própria)
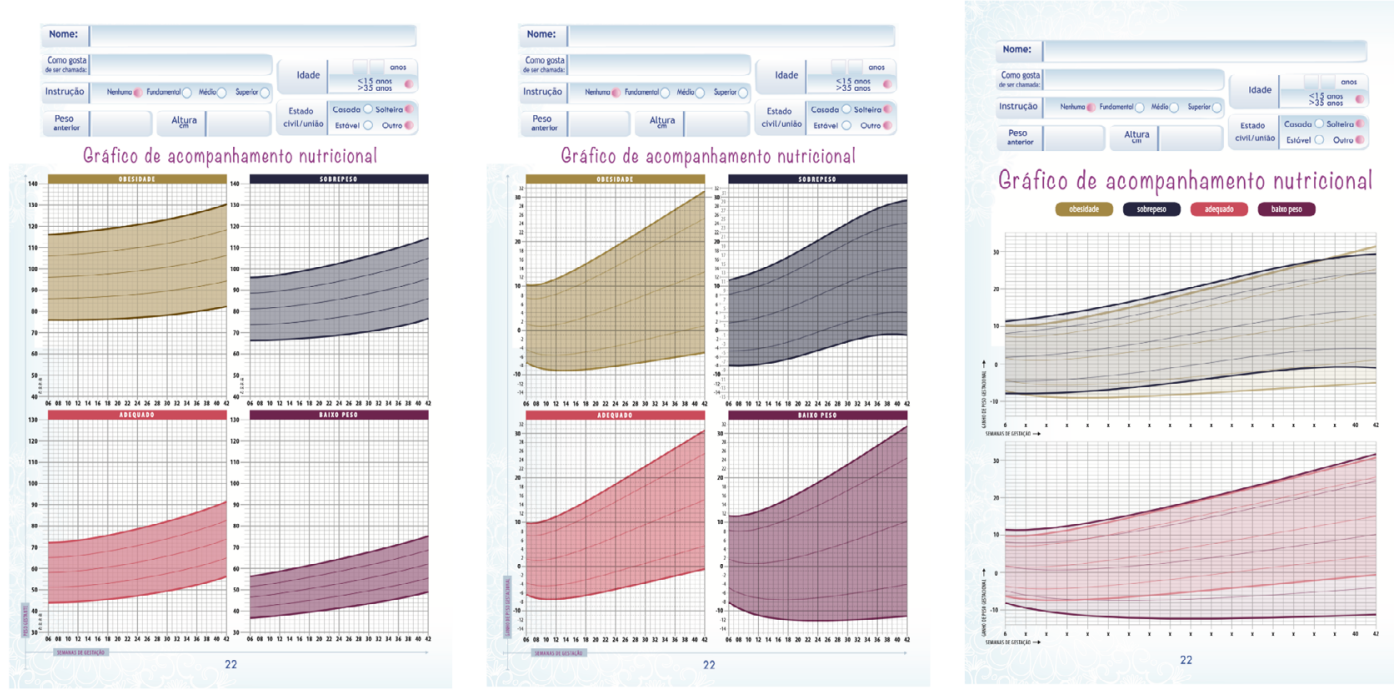

Ainda para auxiliar os pesquisadores na escolha sobre entre as três diferentes formas de coleta dos dados do peso da gestante, a equipe de designers desenvolveu o protótipo das curvas alterando o eixo vertical para i) Peso Atual da Gestante e ii) Ganho de Peso em Relação à Última Consulta. Essa também era uma decisão essencial a ser tomada logo nessas fases iniciais de testes pois cada uma dessas formas estabelecia os limites e restrições passíveis de serem exploradas graficamente.

A partir das discussões, julgou-se que i) o eixo vertical deveria registrar o ganho de peso da gestante a partir da última consulta, ii) que as semanas do eixo horizontal deveriam apresentar um range de seis a 41 semanas e que iii) o ideal seria que as curvas se apresentassem sem sobreposição com outras faixas de peso.

Embora os pesquisadores entendessem que essas eram as escolhas ideais para a visualização, ambos os grupos concordaram que essa não deveria ser uma decisão tomada sem a opinião de especialistas na área da saúde e dos profissionais que atendem as gestantes nos sistemas de saúde. Para incorporar o conhecimento de tais stakeholders, foram programados dois workshops de validação, a serem descritos nas próximas seções. 


\section{$31^{\circ}$ Workshop de avaliação das curvas}

A Oficina de trabalho - Curvas de Ganho de Peso Gestacional foi um workshop realizado em Brasília, no Distrito Federal, com 25 especialistas de diversas áreas técnicas do Ministério da Saúde, tais como a Coordenação Geral de Alimentação e Nutrição (CGAN), Sistema Vigilância Alimentar e Nutricional (SISVAN) além de analistas de outras instituições interessadas no sucesso da ferramenta, como a Organização Pan-Americana de Saúde (OPAS).

Em um primeiro momento, foi apresentada a metodologia utilizada para o cálculo das novas curvas, esclarecendo os dados levados em consideração, quais foram as dificuldades relacionadas à limpeza e harmonização dos dados e desafios estatísticos que se revelaram ao longo do processo. Em seguida, foram estruturadas cinco dinâmicas, com seus objetivos e perguntas a serem respondidas. A quarta dinâmica dizia respeito ao novo design da proposta, considerando a seguinte questão: dado o espaço existente na caderneta, como é possível representar as curvas de maneiras distintas, equilibrando tamanho das curvas e das informações de texto, sobreposição das curvas e legibilidade/marcação? Quais são as possibilidades de apresentações e elementos gráficos que permitem o preenchimento das informações da mãe na caderneta?

Para responder tais questões, foram preparados kits impressos com diversos estudos relacionados, totalizando seis folhas por kit. A primeira folha apresentava um gráfico com as quatro curvas sobrepostas, tendo como eixo vertical o ganho de peso desde a última medição. A segunda folha, apresentava dois gráficos, com cada gráfico sobrepondo duas faixas de peso distintas. A terceira folha, apresentava quatro gráficos, cada um dedicado a uma faixa de peso. A quarta e a quinta folhas apresentavam a mesma estrutura da terceira, com a diferença que uma apresentava o peso da gestante no eixo vertical e a quinta apresentava o IMC da gestante. Por fim, a sexta folha apresentava seis gráficos com explorações gráficas relacionadas às diferentes formas de preencher os dados (figura 7 e 8 , já apresentadas anteriormente).

Os participantes foram divididos em grupos e cada um dos grupos recebeu um kit impresso com dados de uma gestação e instruções sobre a forma de preenchimento. A eles foi dado um tempo para preencher a curva com tais dados e discutir entre si sobre as vantagens $\mathrm{e}$ desvantagens do uso de cada uma das propostas e, posteriormente, expor seus comentários a todos, respondendo qual das versões era a ideal e a justificativa para tal escolha.

A maioria dos grupos concordou que a versão da curva com a informação do ganho de peso desde a última consulta era uma alternativa melhor que o IMC ou o peso da gestante para figurar no eixo vertical do gráfico, tendo em vista a granularidade possível. Também ficou clara a necessidade de que as curvas fossem separadas para facilitar a legibilidade das informações e evitar ambiguidades na leitura e no preenchimento dos dados. Um dos técnicos presentes no evento entendeu a importância dessas restrições e se prontificou a levar essa discussão a outras instâncias do MS para negociar a possibilidade de inserir mais uma folha na caderneta da gestante e disponibilizar mais espaço para as quatro categorias da ferramenta. A partir daí, os designers começaram a trabalhar em dois cenários: no melhor deles, cada uma das 
categorias teria sua própria curva em uma página inteira e, no pior, duas categorias por página, totalizando duas páginas na caderneta da gestante.

Outro aspecto interessante que surgiu em meio às discussões foi a necessidade de o gráfico da curva suportar a reprodução em máquinas de xerox ou em mimeógrafos. Segundo uma das participantes da oficina, é comum que pequenas cidades do interior do Brasil recebam um número reduzido de cadernetas da gestante, o que força os profissionais de saúde dessas localidades a multiplicarem as cadernetas a partir desses processos de reprodução. Nesse sentido, os designers realizaram ajustes na espessura das linhas, tamanho das tipografias e testes de impressão utilizando tais equipamentos para atestar a resistência e legibilidade das informações.

\section{Formulário online de avaliação das curvas}

Segundo o planejamento inicial, o passo seguinte ao seminário de avaliação no MS seria o de uma nova bateria de avaliações in loco, dessa vez com profissionais de saúde que utilizam a ferramenta atual como parte do trabalho de acompanhamento da gestação. No entanto, devido às dificuldades impostas pela pandemia de COVID-19, foi necessária uma adaptação na atividade: o grupo de pesquisadores construiu um formulário online com questões que discutiam as novas páginas propostas, considerando debates relacionados aos elementos gráficos das curvas, compreensão da nova visualização e possíveis dificuldades ou apontamentos sobre o preenchimento dela. A partir das observações coletadas, foram realizados ajustes nos espaçamentos, no tamanho e no peso das fontes e na demarcação dos trimestres de gestação.

\section{Versão atual impressa das novas curvas}

Realizadas as duas atividades de validação dos gráficos, a primeira versão das curvas se estabeleceu. A figura 9 representa o modelo final do gráfico da curva, estruturado após as validações descritas na seção anterior. 
Figura 9 - Gráfico final das curvas de ganho de peso - categoria Eutrofia (Fonte: autoria própria)

EUTROFIA (IMC $\left.\geq 18,5 \mathrm{~kg} / \mathrm{m}^{2} \mathrm{e}<25 \mathrm{~kg} / \mathrm{m}^{2}\right)$

10111213141516171819202122232425262728293031323334353637383940

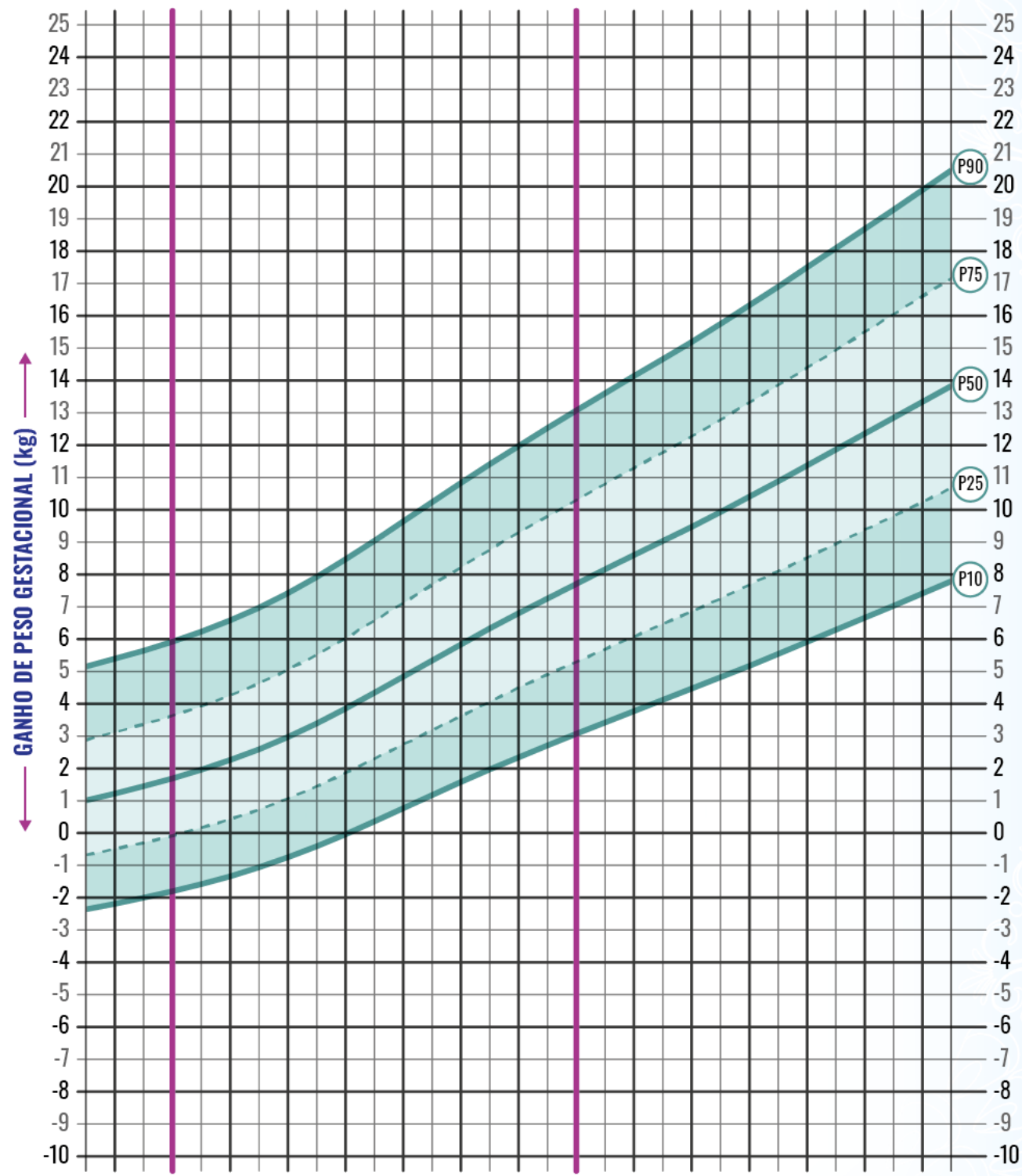

10111213141516171819202122232425262728293031323334353637383940

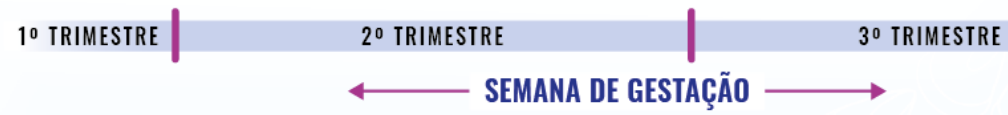

No topo da página, o título informa sobre qual das quatro categorias de IMC a curva de ganho de peso se refere. Foi decidido que todos os numerais dos eixos deveriam ser impressos. Para auxiliar a leitura destes, decidiu-se por intercalar a opacidade dos valores e das linhas em ambos os eixos. Além disso, os numerais foram colocados em ambos os lados, para facilitar a inserção das medições ao longo do tempo. Conforme a solicitação dos profissionais de saúde, as semanas 13 e 27 foram destacadas para demarcar os três semestres da gestação. Cada uma das curvas é identificada por uma cor distinta. Além disso, 
aproveitando que as curvas ganharam páginas próprias, aproveitou-se o espaço anteriormente utilizado para inserção das instruções sobre leitura e preenchimento dos dados, como pode ser visto na figura 10 .

Figura 10 - Conjunto de páginas para a caderneta da Gestante (Fonte: autoria própria)
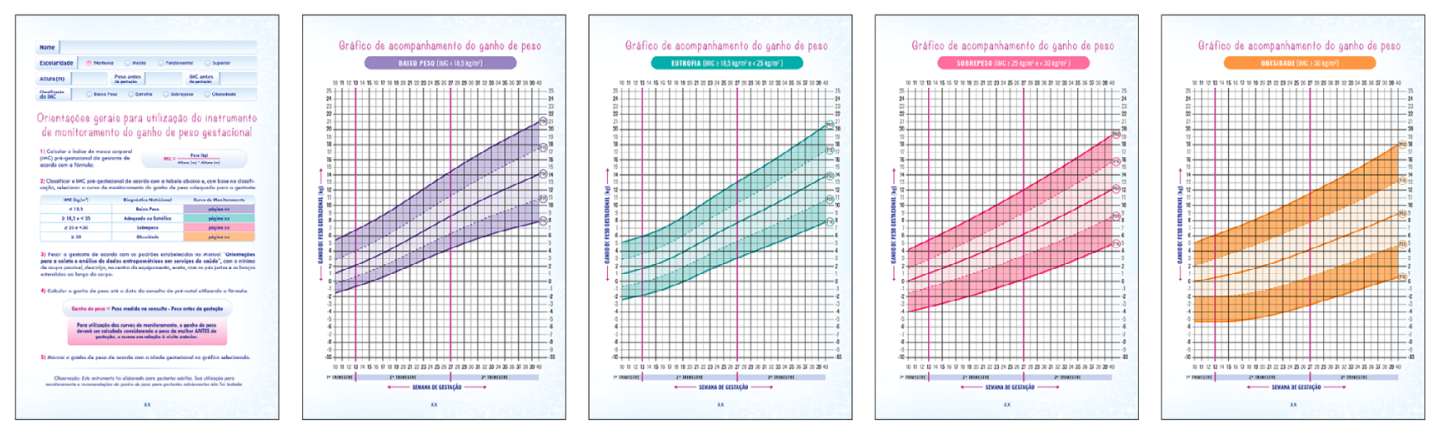

Até o momento da submissão do presente artigo, a implantação das curvas na caderneta ainda se encontrava em processo de desenvolvimento junto ao MS.

\section{Versão atual digital das novas curvas}

O primeiro protótipo desenvolvido até o momento segue as mesmas lógicas da curva impressa, porém com possibilidades adicionais de interação. Primeiramente, o usuário insere a data aproximada do primeiro dia da gestação, além do peso e altura da mãe. Com essas informações, o sistema calcula automaticamente qual das curvas de ganho de a gestante deve seguir para um ganho de peso saudável de acordo com o seu corpo. Em seguida, é possível inserir os dados das consultas seguintes - i) data da consulta e ii) peso da gestante no momento da consulta -, que são sinalizados na curva por um ponto de cor escura. Esses pontos se conectam, desenhando a curva de ganho de peso da mãe que pode ser comparada com a curva da categoria estabelecida no primeiro passo. A figura 11 apresenta um exemplo de uso da ferramenta. 
Figura 11 - Curvas digitais (Fonte: autoria própria)

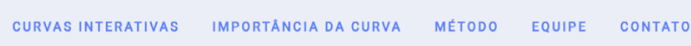

\section{Dados da gestante}

IMC $=22.9 \mathrm{~kg} / \mathrm{m}^{2}$

Categoria = Eutrofia

$6^{\mathrm{a}}$ consulta

Idade gestacional $=26$ semanas

Ganho de peso gestacional $=6.0 \mathrm{~kg}$

Acompanhamento

data da consulta

09/08/2021

$\mathrm{dd} / \mathrm{mm} / \mathrm{aaaa}$

peso na consulta

61

em kg

LIMPAR ADICIONAR

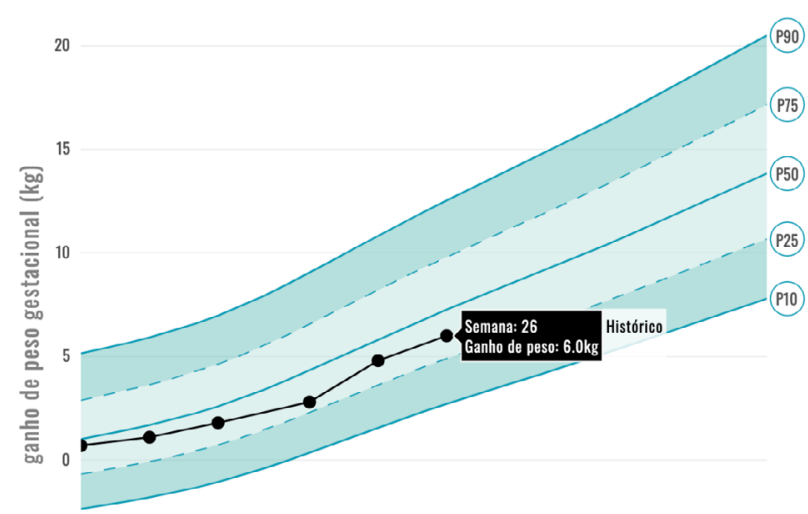

$-5$

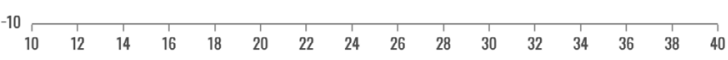

semana de gestação

histórico de consultas

\begin{tabular}{|c|c|c|c|c|}
\hline Data da consulta & Semana de gestaçäa & Peso na consulta $(\mathrm{kg}) \triangleq$ & Ganho de peso $(\mathbf{k g}) \triangleq$ & \\
\hline $02 / 08 / 2021$ & 26,0 & 61,0 & 6,0 & $\mathrm{x}$ \\
\hline $12 / \theta 7 / 2 \theta 21$ & $23, \theta$ & 59,8 & 4,8 & $\mathrm{x}$ \\
\hline $21 / 06 / 2021$ & $2 \theta, \theta$ & 57,8 & 2,8 & $\mathrm{x}$ \\
\hline $24 / 05 / 2021$ & 16,0 & 56,8 & 1,8 & $\mathrm{x}$ \\
\hline$\theta 3 / 05 / 2 \theta 21$ & $13, \theta$ & 56,1 & 1,1 & $\mathrm{x}$ \\
\hline
\end{tabular}

A versão online foi desenvolvida utilizando a linguagem $R$ e o pacote Shiny, tecnologias amplamente utilizadas por acadêmicos devido a sua facilidade de manutenção, documentação disponível e reprodutibilidade. Todo o código do protótipo foi repassado aos pesquisadores, para que eles tivessem controle da tecnologia e pudessem ajustar os dados dos cálculos das curvas sem depender da equipe de designers.

Finalizada a descrição do desenvolvimento do projeto, passamos à seção de discussão.

\section{Discussão}

Ao refletir sobre os processos de design das soluções gráficas propostas, elencamos três tópicos que sintetizam os aprendizados adquiridos ao longo do trabalho:

\section{i) Explorar o design de informação e o desenvolvimento de protótipos enquanto meio de} democratização da ciência: o conhecimento em design se abriga no designer, nos artefatos desenvolvidos e nos processos utilizados (Cross, 1999). Tanto a versão impressa quanto a 
digital das novas curvas auxiliam profissionais de saúde e gestantes a se comunicarem melhor por meio de uma ferramenta que incorpora conhecimentos sobre análise e visualização de dados. Os protótipos construídos serviram para estabelecer territórios comuns para a emergência do diálogo, auxiliando as pessoas envolvidas no processo de construção nas reflexões e nos debates que embasaram as decisões tomadas ao longo do projeto. A representação das curvas sobrepostas, por exemplo, iniciou um debate sobre a importância do cuidado e da atenção para com os diferentes tipos de corpos das mulheres. Quando se discute sobre a inclusão por meio dos dados, isso não se relaciona apenas com as bases de dados coletadas, mas também com a representação visual advinda dessas informações: independentemente do seu peso, todas as gestantes devem ter acesso ao cuidado pré-natal.

ii) Amplificar o acesso das curvas por meio de novos formatos: entende-se que a versão online das curvas é uma abordagem inovadora. Ainda que o acesso a internet não seja universal em diversos países em países de baixos e média renda (LMICs) ${ }^{4}$, a disponibilidade das curvas em meios digitais permite que tanto gestantes sem acesso à versão impressa quanto pesquisadores interessados nos estudos possam avaliar o trabalho desenvolvido. O que à primeira vista parece simples, incorpora anos de esforço em pesquisa envolvendo grandes volumes de dados, ciência e tecnologia.

iii) Assumir a importância de processos de co-design: a versão atual da caderneta dispõe de apenas uma página para a ferramenta de acompanhamento do ganho de peso, mas, após reuniões com as áreas técnicas do MS, compreendeu-se a importância da ferramenta e a necessidade de mais espaço para que todas as curvas pudessem ser compreendidas. À primeira vista, essa parece uma ação trivial. Contudo, ao considerar que a caderneta da Gestante é impressa e distribuída a todo Brasil, uma simples mudança como essa pode acarretar desafios financeiros e até mesmo políticos. Envolver tal miríade de gestores, técnicos e profissionais de saúde no processo de design é laborioso, mas necessário, pois incorpora pontos de vista distintos e extremamente importantes para o bom uso e adoção da nova proposta.

\section{Considerações Finais}

Traduzir e simplificar toda riqueza dos dados em uma visualização gráfica foi um grande desafio. Desde a etapa de compreensão da complexidade das curvas até o desenvolvimento da última versão preparada para a caderneta do SUS, foram necessárias mais de 100 diferentes explorações gráficas que serviram para alinhar as expectativas da equipe de design, equipe de pesquisa e de técnicas e técnicos do MS e da atenção básica de saúde que iria utilizar o instrumento.

\footnotetext{
${ }^{4}$ Disponível em <https://data. worldbank.org/indicator/IT.NET.USER.ZS?end=2017\&locations=XO\&start=2017\& view=map $>$. Acesso em 9 de maio de 2021.
} 
A versão final da visualização considerou estudos relacionados aos diferentes tipos de daltonismo, cópia por máquinas de xerox, análise de granularidade, compreensão dos usuários sobre variáveis de acompanhamento de peso da gestante e o seu uso em diferentes contextos do SUS. Estudos futuros devem dar conta de acompanhar o uso da ferramenta após a sua implementação nos sistemas de saúde, identificando pontos de melhoria conforme o modelo se estabelece ao longo do tempo e avaliação de novos ajustes a serem realizados.

Com a criação das versões impressa e digital da ferramenta, as representações gráficas das curvas incorporam a premissa de que todas as mães brasileiras têm direito a uma ciência aberta, acessível e acurada. Entende-se, também, que esse é um projeto de inclusão por meio dos dados que se preocupa com a melhoria da saúde das mães e de recém-nascidos e com a democratização da ciência de dados no país.

\section{Agradecimento}

Os autores deste artigo agradecem a Gilberto Kac e Thaís Rangel e a todo o time de pesquisadoras e pesquisadores pelo trabalho científico de excelência, pela paciência e pela parceria ao longo do projeto; ao time de profissionais da odd.studio, pelo apoio vital no desenvolvimento dos gráficos impressos e digitais, especialmente à Ana Paula Bertol e Gabriel Antônio Rezende de Paula pela atenção e cuidado dedicados à revisão do texto; por fim, agradecemos à todas as técnicas e técnicos do Ministério da Saúde e de outras diversas instituições envolvidas nos processos de colaboração para avaliação e melhoria da proposta.

\section{Referências}

Atalah E., Castillo C., Castro R., Aldea A. (1997) Proposal of a new standard for the nutritional assessment of pregnant women. Rev Med Chil ;125(12):1429-36.

Carrilho, T.R.B., Farias, D.R., Batalha, M.A. et al. (2020) Brazilian Maternal and Child Nutrition Consortium: establishment, data harmonization and basic characteristics. Sci Rep 10, 14869. https://doi.org/10.1038/s41598-020-71612-8

Cross, N. (1999). Design Research: A Disciplined Conversation. Design Issues, 15(2), 5-10. doi: $10.2307 / 1511837$

Gaver, W. (2012). What Should We Expect From Research Through Design?. Conference on Human Factors in Computing Systems - Proceedings. https://dx.doi.org/10.1145/2207676.2208538

Kac, G., Nucci, L., Spyrides, M., Duncan B., Schmidt M. (2009) Evaluation of the ability of a Latin-American gestational weight curve to predict adverse pregnancy outcomes. Int $J$ Gynecol Obstet;106(3): 223-6.

Kac, G., Carilho, T., Rasmussen, K. M., Reichenheim, M. E., Farias, D. R., Hutcheon, J. A., \& Brazilian Maternal and Child Nutrition Consortium (2021). Gestational weight gain charts: results from the Brazilian Maternal and Child Nutrition Consortium. The American journal of clinical nutrition, 113(5), 1351-1360. https://doi.org/10.1093/ajcn/nqaa402 
Koskinen, I., Zimmerman, J., Binder, T., Redstrom, J., \& Wensveen, S. (2011) Design research through practice: From the lab, field, and showroom. Elsevier, Amsterdam, NL.

Rasmussen, K. M., Yaktine, A. L., \& Institute of Medicine (US) and National Research Council (US) Committee to Reexamine IOM Pregnancy Weight Guidelines (Eds.). (2009). Weight Gain During Pregnancy: Reexamining the Guidelines. National Academies Press (US). doi:10.17226/12584

Sanders, E., Stappers, P. (2008) Co-creation and the new landscapes of design, CoDesign, 4:1, 5-18, DOI: 10.1080/15710880701875068

\section{Sobre o(a/s) autor(a/es)}

Bruno Augusto Lorenz, Me., odd.studio, Brasil <bruno@odd.studio>

Letícia Ange Pozza, Me., odd.studio, Brasil<leticia@odd.studio>

Thaís Rangel Bousquet Carrilho, Me., UFRJ, Brasil <thaisrangelnut@gmail.com>

Gilberto Kac, PhD, UFRJ, Brasil <gkac@nutricao.ufrj.br>

Francisco Estivallet, B.Sc, odd.studio, Brasil <francisco@odd.studio> 